\title{
BMJ Open Quantifying healthcare and welfare sector workers' preferences around COVID-19 vaccination: a cross-sectional, single-profile discrete-choice experiment in France
}

Carolina Díaz Luévano, ${ }^{1}$ Jonathan Sicsic, ${ }^{2}$ Gerard Pellissier, ${ }^{3}$ Sandra Chyderiotis, ${ }^{4}$ Pierre Arwidson, ${ }^{5}$ Cyril Olivier, ${ }^{3}$ Amandine Gagneux-Brunon, ${ }^{6,7}$ Elisabeth Botelho-Nevers, ${ }^{6,7}$ Elisabeth Bouvet, ${ }^{3}$ Judith Mueller (1) ${ }^{1,4}$

To cite: Díaz Luévano C, Sicsic J, Pellissier G, et al. Quantifying healthcare and welfare sector workers' preferences around COVID-19 vaccination: a cross-sectional, single-profile discrete-choice experiment in France. BMJ Open 2021;11:e055148. doi:10.1136/ bmjopen-2021-055148

- Prepublication history and additional supplemental material for this paper are available online. To view these files, please visit the journal online (http://dx.doi.org/10.1136/ bmjopen-2021-055148).

Received 05 July 2021 Accepted 19 September 2021

Check for updates

(c) Author(s) (or their employer(s)) 2021. Re-use permitted under CC BY-NC. No commercial re-use. See rights and permissions. Published by BMJ.

For numbered affiliations see end of article.

Correspondence to Dr Judith Mueller; judith.mueller@ehesp.fr

\section{ABSTRACT}

Objectives To analyse preferences around promotion of COVID-19 vaccination among workers in the healthcare and welfare sector in Fance at the start of the vaccination campaign.

Design Single-profile discrete-choice experiment. Respondents in three random blocks chose between accepting or rejecting eight hypothetical COVID-19 vaccination scenarios.

Setting 4346 healthcare and welfare sector workers in France, recruited through nation-wide snowball sampling, December 2020 to January 2021.

Outcome The primary outcomes were the effects of attributes' levels on hypothetical acceptance, expressed as ORs relative to the reference level. The secondary outcome was vaccine eagerness as certainty of decision, ranging from -10 to +10 .

Results Among all participants, $61.1 \%$ made uniform decisions, including $17.2 \%$ always refusing vaccination across all scenarios (serial non-demanders). Among 1691 respondents making variable decisions, a strong negative impact on acceptance was observed with $50 \%$ vaccine efficacy (compared with $90 \%$ efficacy: OR $0.05,95 \% \mathrm{Cl}$ 0.04 to 0.06 ) and the mention of a positive benefit-risk balance (compared with absence of severe and frequent side effects: OR $0.40,0.34$ to 0.46 ). The highest positive impact was the prospect of safely meeting older people and contributing to epidemic control (compared with no indirect protection: OR 4.10, 3.49 to 4.82 and 2.87, 2.34 to 3.50 , respectively). Predicted acceptance was $93.8 \%$ for optimised communication on messenger RNA vaccines and $16.0 \%$ for vector-based vaccines recommended to $\geq 55$-year-old persons. Vaccine eagerness among serial non-demanders slightly but significantly increased with the prospect of safely meeting older people and epidemic control and reduced with lower vaccine efficacy.

Discussion Vaccine promotion towards healthcare and welfare sector workers who hesitate or refuse vaccination should avoid the notion of benefit-risk balance, while collective benefit communication with personal utility can lever acceptance. Vaccines with limited efficacy will unlikely achieve high uptake.
Strengths and limitations of this study

- This single-profile discrete-choice experiment (DCE) conducted a realistic evaluation of COVID-19 vaccine preferences among a large sample of healthcare and welfare sector workers in France, covering a large range of professional profiles.

- The DCE focused on communication content that could optimise vaccine promotion to vaccinehesitant healthcare and welfare workers.

- Based on choice certainty, the approach of vaccine eagerness aimed at gaining insight into preferences among healthcare and welfare workers constantly refusing vaccination.

- DCEs evaluate hypothetical, not actual, vaccine acceptance and preferences may evolve over time.

\section{INTRODUCTION}

In the current COVID-19 epidemic, healthcare workers (HCWs) are at increased risk of infection and disease as well as transmitting the virus to patients, colleagues and their personal environment. Most countries have prioritised HCWs COVID-19 vaccination. Among HCWs in France, the seasonal influenza vaccine coverage has been as low as $35 \%$ for more than a decade, ${ }^{12}$ due to complacency and lack of confidence for some and lack of convenience for others. ${ }^{3}$ At the beginning of the COVID-19 vaccination campaign among HCWs, there was concern that this vaccine hesitancy may also apply to COVID-19. In July 2021, the COVID-19 vaccine coverage for at least one dose among HCW in France was estimated at $60.5 \%$ and $80.5 \%$ in nursing homes and private practice, respectively. ${ }^{4}$ At the same time, a strong gradient from medical professions $(76 \%)$ to nurses $(62 \%)$ and nurse assistants (55\%) was described in 
hospitals, ${ }^{5}$ similar to the gradient in vaccine intention observed 1 year earlier. ${ }^{6}$ A COVID-19 vaccine mandate for healthcare and welfare sector workers (HWSW) in France entered into force in September 2021. To prepare for theoretical future revaccination and learn for future epidemic vaccination campaigns, it is important to understand which characteristics of the vaccine and vaccination context can make hesitant HWSWs decide in favour of COVID-19 vaccination and to optimise communication messages accordingly.

There is a substantial body of evidence on factors impacting HCWs' acceptance of COVID-19 vaccination and vaccine hesitancy. ${ }^{7}$ Vaccine hesitancy is described as a continuum between complete acceptance and refusal. ${ }^{8}$ Discrete-choice experiments (DCEs) have been increasingly used to study preferences around COVID-19 vaccines and predict uptake ${ }^{9-14}$ focusing on fixed vaccine characteristics such as efficacy, duration of protection and risk of side effects. However, few studies have assessed the effect of contextual, ${ }^{10121516}$ interindividual $^{131516}$ and communication factors on the willingness to receive COVID-19 vaccine. To our knowledge, only one unpublished report ${ }^{13}$ has focused on HCWs, suggesting that vaccine intention was positively associated with acceptance among (the) social contacts, in addition to vaccine effectiveness and safety, risk of disease and out-of-pocket payment.

In the present DCE study, we aimed at quantifying preferences around COVID-19 vaccination among HWSWs in France, to understand incomplete vaccine uptake and identify targeted communication strategies, in particular, among hesitating persons and on issues that will continue to be subjected to scientific uncertainty for upcoming vaccines, such as vaccine capacity to block transmission, duration of vaccine protection and vaccine safety.

\section{METHODS}

\section{Study design and participant inclusion}

We conducted a cross-sectional study among HWSWs in France, including a single-profile DCE. Between 18 December 2020 through 1 February 2021, the Research Group for the Prevention of Occupational Infections in Healthcare Workers (GERES) published an online questionnaire on the Sphinx online survey platform, which was disseminated throughout France by different HCW networks. Through the 'snowball sampling' effect, the questionnaire reached a total of 9580 participants working in hospitals, nursing homes and other welfare institutions and private practice. This comprised diverse healthcare-related and welfare-related careers, including physicians, nurses, nurse assistants, other paramedical professionals and also social workers, administrative and logistic staff. Since participants forwarded the questionnaire across their own networks, response rate could not be estimated. No exact data exist on the total number of workers in the healthcare and welfare sectors in France.
Official estimates report 661000 nurses, 390000 nurse assistants, around 175000 other paramedical professionals and about 362000 biomedical professionals (data source: https://www.ars.sante.fr/media/23043/download). We aimed at a minimal size of 200 per strata of professional group, according to the guidelines for conjoint analyses. ${ }^{17}$ Because DCEs are exploratory by nature and estimate multiple parameters (the various attribute levels), we did not calculate a maximal sample size, but maximised the number of responses, to be able to stratify analyses with additional participant characteristics. At study start, vaccine efficacy data had been published or announced by AstraZeneca, Pfizer and Moderna. In France, vaccination in nursing homes (Pfizer/BioNTech vaccine) had started late December 2020, while vaccination for $\geq 50$-year-old HCWs started on 4 January 2021. The vaccine campaign targeting persons $\geq 75$-year-old persons was launched starting 15 January 2021 (using Pfizer/BioNTech and Moderna vaccines). During the entire study period, no travel or work restrictions were imposed, but a curfew was in place 20:00-06:00, which was extended to 18:00-06:00 starting 15 January 2021, as well.

\section{Questionnaire}

The first part of the survey collected sociodemographic, professional and health-related characteristics of participants, including previous vaccination against seasonal influenza and intention to accept COVID-19 vaccine. Participants were then pseudorandomly directed (based on a choice between square and circle) to one of the two questionnaires of the second part: either a standardised questionnaire on knowledge and attitudes, regarding COVID-19 vaccination, or the DCE presented in this report. Anticipated and effective survey completion took approximately 10 min. To ensure complete survey responses in order to fully analyse preferences, all choice tasks of the DCE were mandatory.

\section{Design of the single profile DCE}

We designed the DCE tool with five attributes (efficacy, indirect protection, safety, protection duration, recommendation/incentive source) and according to levels (table 1), based on a literature review on vaccine intention and vaccine-related preferences among HCWs,${ }^{18}$ COVID-19 vaccine intention available for the general population ${ }^{19}$ and a study on COVID-19 vaccine acceptance conducted during summer 2020 among HCWs in France. ${ }^{3}$ The tool was designed as a singleprofile DCE, which implies that participants did not choose between two scenarios as in classical DCE, ${ }^{20}$ but made binary decisions for or against accepting immediate vaccination presented through a series of imaginary scenarios (choice tasks) with varying attribute levels. This approach has been used for previous DCE studies on vaccination decisions (ConjointVac studies), ${ }^{18} 21$ because it is more appropriate to study the effect of inter-individual or contextual factors on 
Table 1 Attributes and levels with hypotheses on the expected effects (OR relative to the reference level)

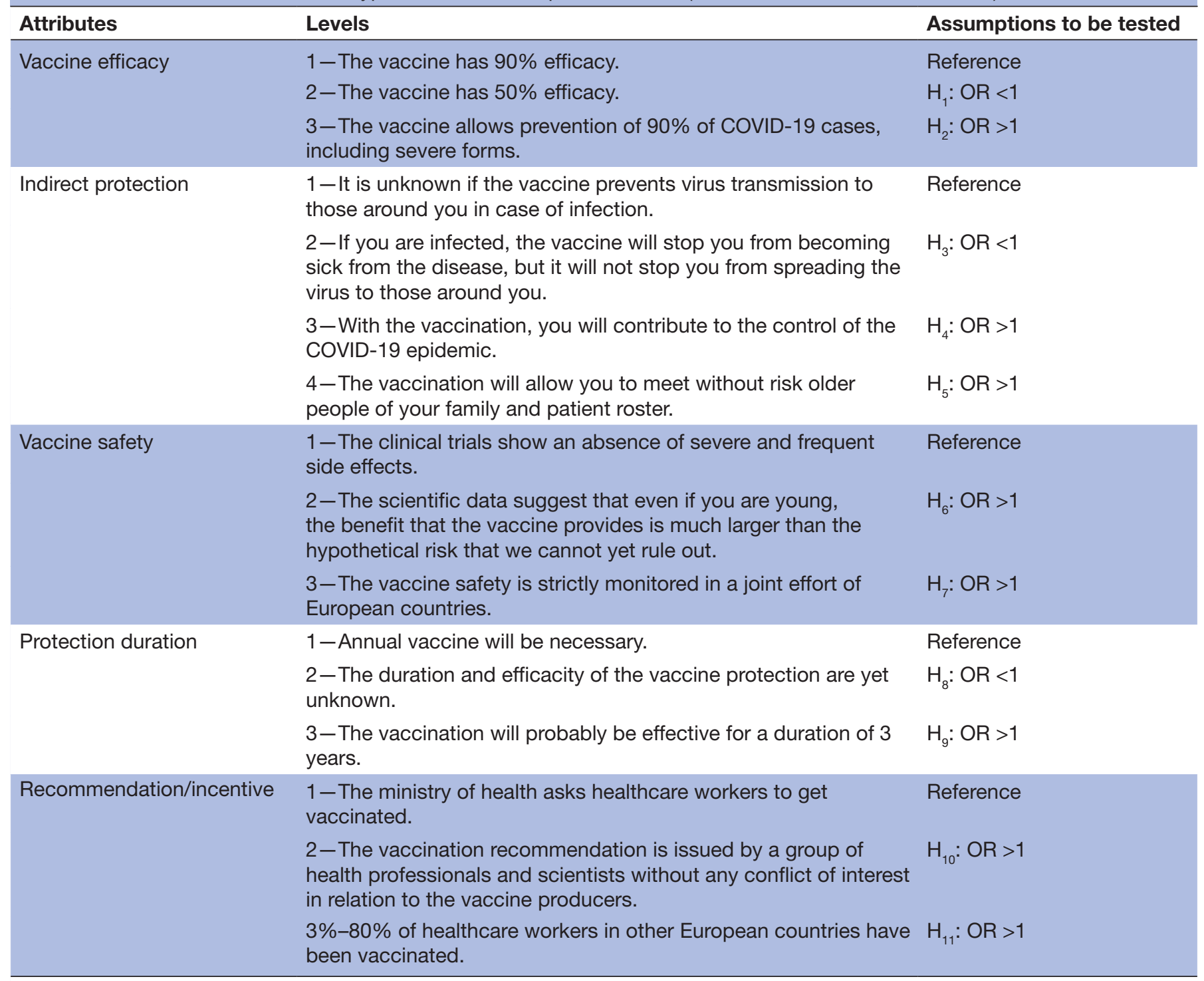

$\mathrm{H}$, hypothesis.

choice. Immediately after the choice question, respondents were asked to self-report their level of certitude on a $0-10$ scale (figure 1 ).

The full-factorial design encompassing all five attributes with their corresponding variables resulted in 324 different combinations, from which we selected 24 scenarios for an efficient design (based on a priori assumptions regarding the direction of the effects, table 1) using NGENE software (Choice metrics). The set of 24 scenarios were blocked into three versions of eight scenarios to which participants were pseudorandomly directed (according to the choice of a geometrical figure). We included an interaction term between two attribute levels based on the hypothesis that in the scenario referring to the control of the epidemic, duration of protection beyond 1 year would have lower effect on acceptance. The tool was pilot tested in thinkaloud sessions with HCWs.
Attribute description and assumptions

Vaccine efficacy (3 levels)

We hypothesised that, compared with the information on $90 \%$ vaccine efficacy, $50 \%$ would have a lower hypothetical acceptance, while adding the specification 'including against serious forms of COVID-19' would yield a higher acceptance. We used the second level as the reference (90\% efficacy), since it allowed us to evaluate the effect that a diminution of efficacy (50\% efficacy) would have on the hypothetical vaccine acceptance.

Vaccine safety (3 levels)

It described different types of information regarding the safety of the proposed vaccine. We hypothesised that information on strict safety monitoring in a joint effort of European countries would have a positive effect on hypothetical COVID-19 vaccine acceptance compared with risk negation ('no severe and frequent adverse events in 
You are participating in an information session regarding the vaccine organized for healthcare workers.

Following the information session, you will be able to immediately get vaccinated against COVID-19 at no cost. Below is the information regarding the vaccine.

\begin{tabular}{|c|c|}
\hline Scenario 1 & \\
\hline & $\begin{array}{l}\text { The vaccine allows prevention of } 90 \% \text { of COVID- } 19 \text { cases, including severe } \\
\text { forms } \\
>\text { The vaccination will allow you to meet without risk older people of your family } \\
\text { and patient roster. } \\
>\text { The vaccine safety is strictly monitored in a joint effort of European countries. } \\
>\text { The vaccination will probably be effective for a duration of } 3 \text { years. } \\
>80 \% \text { of healthcare workers in other European countries have been vaccinated. }\end{array}$ \\
\hline
\end{tabular}

Certainty of your decision $(0=$ not at all certain; $10=$ absolutely certain

Figure 1 Example choice task.

clinical trials'), while reference to a benefit-risk balancedespite being a positive message-would reduce it.

Indirect protection (4 levels)

It described different information regarding vaccine impact beyond individual protection. We hypothesised that compared with 'not known yet whether protects against infection,' stating that there was no such indirect protection would decrease acceptance, while reference to control of the epidemic and the possibility to meet elderly friends and family would increase acceptance.

\section{Protection duration (3 levels)}

We hypothesised that, compared with annual vaccination, not knowing the duration of protection would be dissuasive and a duration of 3 years would be motivating.

\section{Recommendation/incentive (3 levels)}

We hypothesised that, compared with a request from the Ministry of Health that HCWs get vaccinated, a recommendation formulated by professionals and researchers without conflict of interest with vaccine manufacturers would be motivating, as would an information-referring to social conformism-that $80 \%$ of HCWs in other European countries have been vaccinated.

\section{Data analysis}

We used bivariate and multivariate models to assess the association between participant characteristics and the frequency of hypothetical vaccine acceptance across scenarios. Variables were included in the final model if they significantly interacted with one or more attributes at the $\mathrm{p}<0.05$ level. We estimated preference weights for each attribute levels using random intercept logit models. Due to the experimental nature of the data, random effects were not significantly different from fixed effects estimates (Hausman test: $\mathrm{p}>0.194$ ).

Using interaction terms, we explored interactions between attributes and participant characteristics as well as between attributes. We report separate preference weight estimates where significant interactions were found.

Uniform respondents were participants who accepted (serial demanders) or refused (serial non-demanders) all eight scenarios. These participants did not contribute to the likelihood of the choice model, but information on decision certainty could be used to retrieve information on their preferences towards vaccination attributes. We created a variable 'vaccine eagerness' based on certainty information, where certainty from refused scenarios was transformed into negative values. Thus, individuals refusing vaccination with a certainty of 10 would have a score of -10 on the vaccine eagerness scale, and those accepting vaccination with a certainty of 10 would have a score of +10 . A negative variation in certainty (eg, decreasing from 10 to 7) among vaccine non-demanders was interpreted as an increase in vaccine eagerness. We used a panel linear regression model to analyse each attribute's impact on vaccine eagerness. Additionally, 
we created a variable for phase of study participation. According to the rollout of the vaccination campaign described above, we defined the three phases of study participation: from 18 December 2020 to 4 January 2021, 5 January to 14 January 2021 and, finally, from 15 January to 1 February 2021.

\section{Predicted acceptance}

For non-uniform respondents, we calculated the predicted acceptance of specific scenarios of practical relevance: (1) optimised communication on messenger RNA (mRNA) vaccines (2) vector-based vaccines recommended to $\geq 55$-year-old persons as currently in place in France and (3) anticipated communication about future vaccines that will have recent licensure and uncertainty around the safety profile and indirect protection. Predicted acceptance was estimated based on the simulation of the utility (assuming linear-in-parameters underlying utility functions) of the respective combination of attribute levels (for scenario $j$ ) using the standard formulae for predictions in logit models:

$$
\text { predicted accept }=1 /\left[1+e^{-\mathrm{utility} j}\right]
$$

Data analysis was performed using Stata/IC V.16.0.

\section{Patient and public involvement}

No patients nor public representatives were involved in the development or conduct of this research.The GERES, as an association focusing on occupational medicine for HCW, represented a main stakeholder and was involved in all steps of the research, including dissemination of results.

\section{RESULTS}

\section{Participant characteristics}

Among the 4346 participants assigned to the DCE (45.4\%), all completed the questionnaire. Sixty-two per cent of the survey participants were younger than 50 years and $76.0 \%$ were woman. Nurses represented $21.9 \%$, nurse assistants and other short-trained HCWs with patient contact $11.2 \%$ and biomedical professions (including physicians, midwives, pharmacists and biologists) $27.0 \%$ of the sample (table 2). Administrative/technical careers accounted for $24.6 \%$. Among physicians in our sample, $62 \%$ were woman and median age group was $35-49$ years compared with $59 \%$ and 49.3 years mean age according to official estimates (data source: French Ministry of Health). Among nurses, $84 \%$ were woman and median age group was 35-49 years (compared with $88 \%$ and 40.2 years mean age).

Those who believed having a risk factor for severe COVID-19 accounted for $18.9 \%$ and $12.5 \%$ reported having been infected with the virus. The percentages indicating influenza vaccination during the 2019-2020 influenza season were $76.1 \%, 51.2 \%$ and $30.4 \%$, respectively, among biomedical professions, nurses and nurse assistants, compared with the national estimates during
2018-2019 of 72.2\%, 35.9\%, 20.9\%, respectively. Participants worked in hospital settings $(61.2 \%)$, nursing homes or other long-term care institutions (16.2\%), ambulatory/seeing patients outside any care institution setting $(15.4 \%)$ or mixed exercise $(6.0 \%)$. All French regions were comprised, including the overseas departments.

\section{Preferences}

Across scenarios, hypothetical vaccination was on average accepted by $60.1 \%$ of participants, ranging from $44.6 \%$ in the least favourable to $82.8 \%$ in the most favourable scenario (online supplemental table 1) and from $55.8 \%$ to $71.9 \%$ and $70.7 \%$ across the three phases of study participation. Uniform respondents comprised $61.1 \%$ of participants $(\mathrm{n}=2655)$, with $43.9 \% \quad(\mathrm{n}=1908)$ always accepting vaccination and $17.2 \%(\mathrm{n}=747)$ always refusing. When removing participants with uniform respondents, vaccination was hypothetically accepted by $55.3 \%$ of participants across all scenarios.

Attribute impacts among participants with nonuniform decisions were all statistically significant, except for recommendation/incentive 'experts without conflict of interest (CoI)' (table 3). The strongest negative impact was observed with vaccine efficacy ' $50 \%$ ' (OR $0.05,95 \%$ CI 0.04 to 0.60$)$, followed by protection duration 'unknown duration' (OR 0.49, 0.42 to 0.57 ) and indirect protection 'individual protection only' (OR $0.47,0.39$ to 0.56 ). The strongest positive impacts were observed for the individual protection attribute: 'meet older people' (OR $4.10,3.48$ to 4.82 ) and 'control of epidemic' (OR 2.87, 2.35 to 3.50 ). Less pronounced but significant impact on vaccine acceptance relative to reference attribute levels was observed for vaccine efficacy ' $90 \%$ including against severe disease' (OR 1.70), '80\% coverage among HCW in other European countries' (OR 1.32) and protection duration 'probably 3 years' (OR 1.19).

Based on marginal effects among the full sample respectively, among non-uniform respondents, vaccine efficacy ' $50 \%$ ' reduced vaccine acceptance by 21 percentage points (respectively, $-38 \mathrm{pp}$ ), while indirect protection 'meet older people' increased it by 7 percentage points (respectively,+23 pp) and 'control of epidemic' by 6 percentage points (respectively, $+17 \mathrm{pp}$ ). Moreover, compared with negating any severe and frequent adverse event, mentioning a positive benefit-risk ratio and 'strictly monitored across the EU (European countries)' both abated vaccine acceptance by 5 percentage points (respectively, $-15 \mathrm{pp}$ ) (figure 2A,B and online supplemental table 2).

\section{Interactions}

Significant interactions with individual characteristics were observed for all attributes (table 4 and online supplemental table 3), in particular, for the attribute vaccine efficacy and characteristics age and professional category. For example, the positive impact of the indirect protection attribute levels 'control of epidemic' was weaker among the 35-year to 49-year-old (OR 2.53) and $\geq 50$-year-old age groups (OR 2.43) than the 18-year 


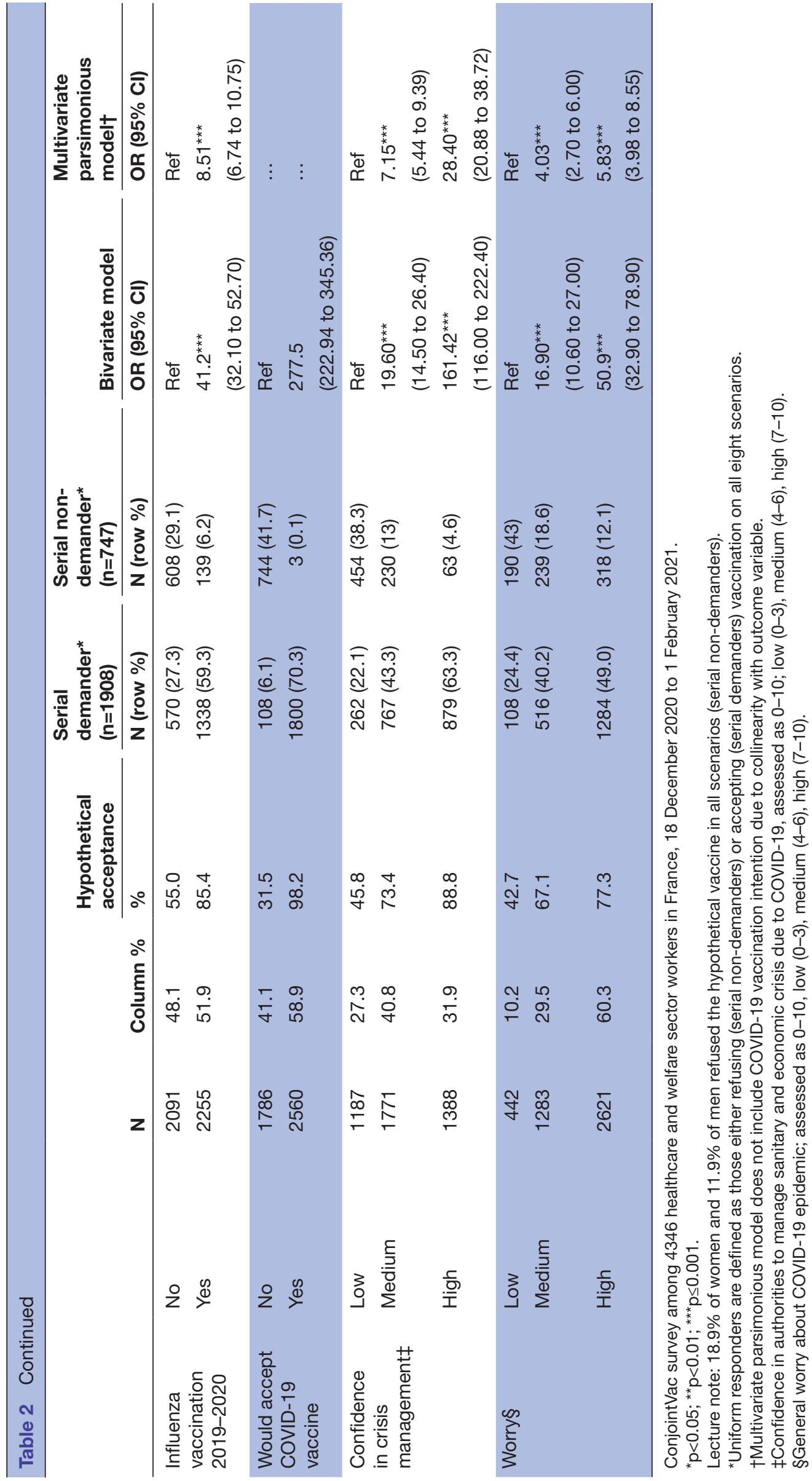




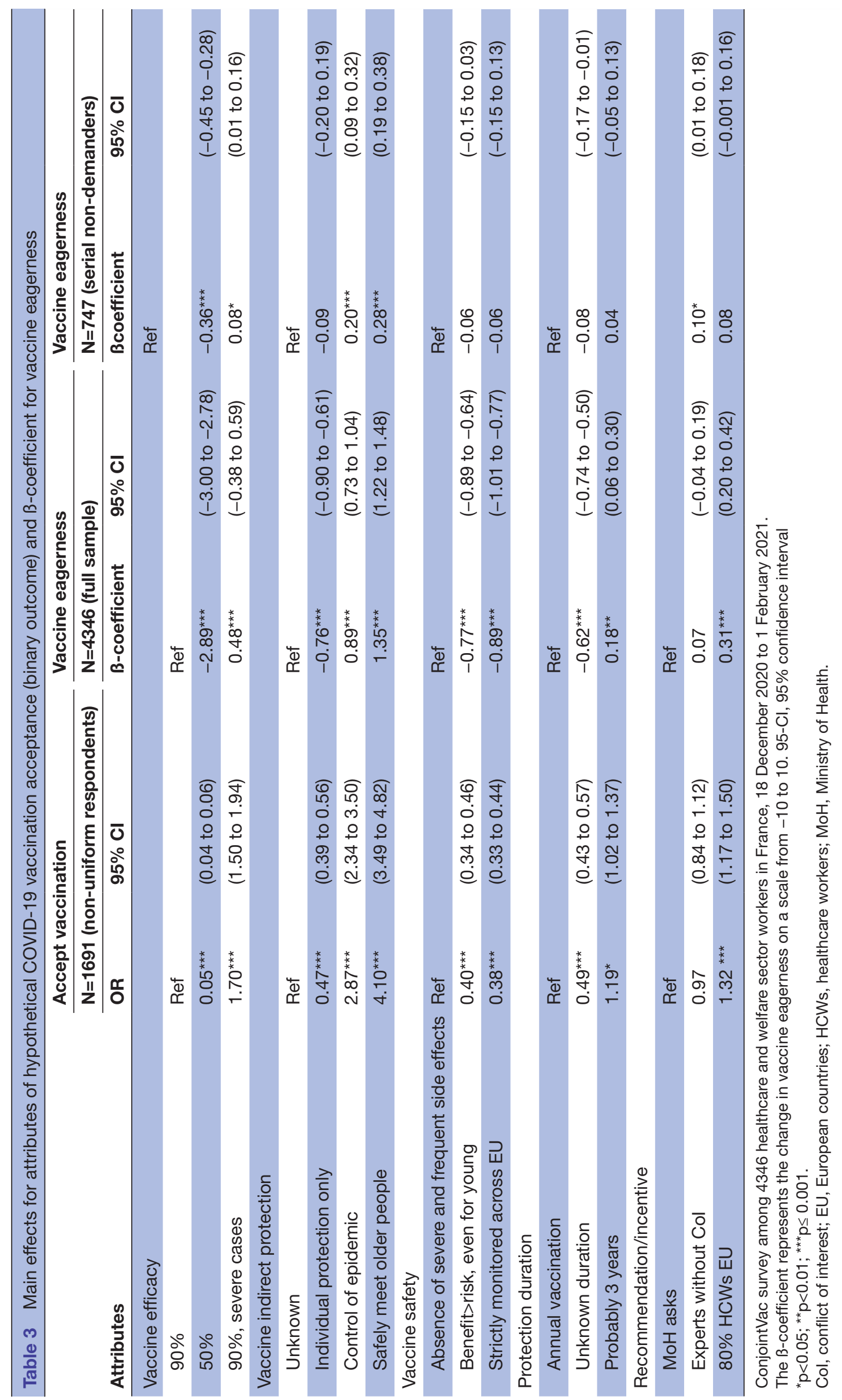


A

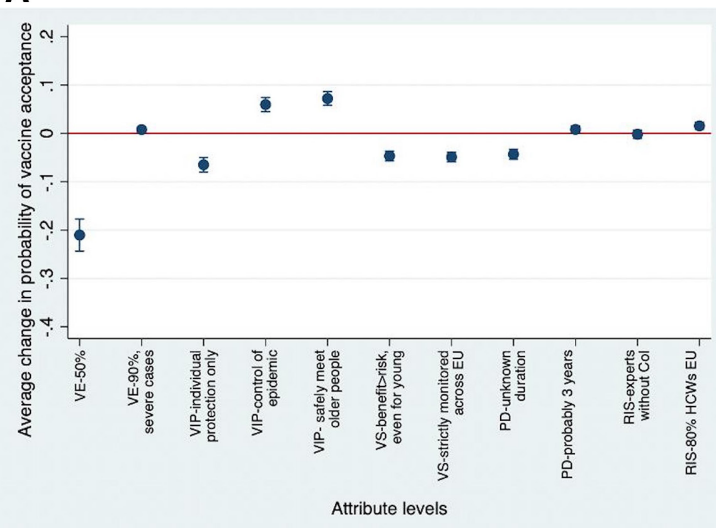

B

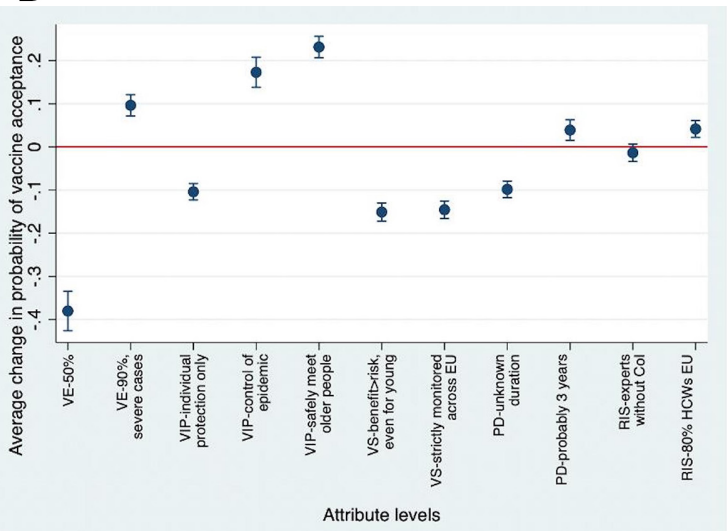

Figure 2 (A and B) Average marginal effects (change in probability of vaccine acceptance) of attribute levels on hypothetical acceptance of vaccination against COVID-19. ConjointVac survey among 4346 healthcare workers in France, 18 December 2020 to 1 February 2021. Attributes: PD, protection duration; RIS, recommendation/incentive; VE, vaccine efficacy; VIP, vaccine indirect protection, VS, vaccine safety. Lecture note: Among all participating HCWs, vaccine efficacy ' $50 \%$ ' instead of ' $90 \%$ ' decreased hypothetical vaccine acceptance by 21 percent points, whereas it decreased by $38 \%$ among non-uniform respondents only. (A) Full sample ( $\mathrm{N}=4346)$. (B) Non-uniform respondents only ( $\mathrm{N}=1691)$. Col, conflict of interest; EU, European countries; HCWs, healthcare workers.

to 34-year-old group (OR 4.77). A similar trend was observed for the level 'meet older people'.

We observed a significantly weaker aversion against the mention of a positive benefit-risk balance among biomedical professions (OR 0.60), compared with nurses (0.35) and other professional categories. Additionally, among nurse assistants, a recommendation by an expert group without conflict of interest had a significant negative impact (OR $0.67,95 \%$ CI 0.42 to 0.95 ). Attribute impacts were relatively stable across the three study phases. Some significant but not substantial changes from phase 1 to 3 were observed (online supplemental table 3 ).

In assessing the interaction between attributes (online supplemental table 4), the positive impact of protection duration 'probably 3 years' increased and became significant in scenarios with indirect protection 'control of epidemic' (OR 1.98, 1.18 to 3.43).

\section{Vaccine eagerness}

Among the full sample of participants, including serial demanders and non-demanders, the average level of vaccine eagerness (on a -10 to 10 scale) in the 24 scenarios ranged from -0.78 to 5.86 (online supplemental table 1 and online supplemental figure 1). Analyses based on vaccine eagerness in the full sample showed a similar pattern of preference weights as the analysis on vaccine acceptance among non-uniform responders, with strongest negative impact from vaccine efficacy ' $50 \%$ ' $(\beta=-2.89$, $95 \% \mathrm{CI}-3.01$ to -2.78 ) and the strongest positive impact from indirect protection 'meet older people' $(B=1.35$, 1.22 to 1.48$)$. Among serial non-demanders, all attribute levels showed a similar direction of effect as in the full sample, but effects were substantially weaker and mostly insignificant (table 3 ). We observed a significant negative impact from vaccine efficacy ' $50 \%$ ' $(\beta=-0.36,-0.45$ to -0.28$)$ and significant positive impacts from indirect protection 'meet older people' $(B=0.28,0.19$ to 0.38$)$ and 'control of epidemic' ( $\beta=0.20,0.09$ to 0.32 ). Moreover, recommendation/incentive 'experts without CoI' had a significant positive impact ( $\beta=0.10,0.01$ to 0.18 ).

\section{Predicted acceptance}

Among participants with non-uniform decisions, the predicted acceptance of specific scenarios was 93.8\% for optimised communication on mRNA vaccines; $5.4 \%$ for vector-based vaccines recommended to $\geq 55$-year-old persons and $65.2 \%$ for future COVID-19 vaccines with recent licensure (online supplemental table 5). For future COVID-19 vaccines with recent licensure, the predicted acceptance varied substantially between professional categories: $58.3 \%$ among nurses, $53.6 \%$ among nurse assistants, $77.7 \%$ among other paramedical professions, $77.1 \%$ among biomedical professions and $58.7 \%$ among administrative and technical careers.

\section{DISCUSSION}

We used an original single-profile DCE to estimate HWSWs preferences and trade-offs between characteristics of COVID-19 vaccination in France. We found that among HWSWs variably accepting or refusing vaccination, a reduced vaccine efficacy had the strongest negative effect on choices, followed by mentioning a positive benefit-risk ratio when communicating about vaccine safety, whereas communication about indirect protection effects (safely meet older people and epidemic control) had the strongest positive effect.

In France, vaccine promotion and delivery largely relies on physicians, while the COVID-19 campaign was rolled out through to a large extent through vaccination centres, without consultations with treating physicians. Nurses and nurse assistants, and other workers in 


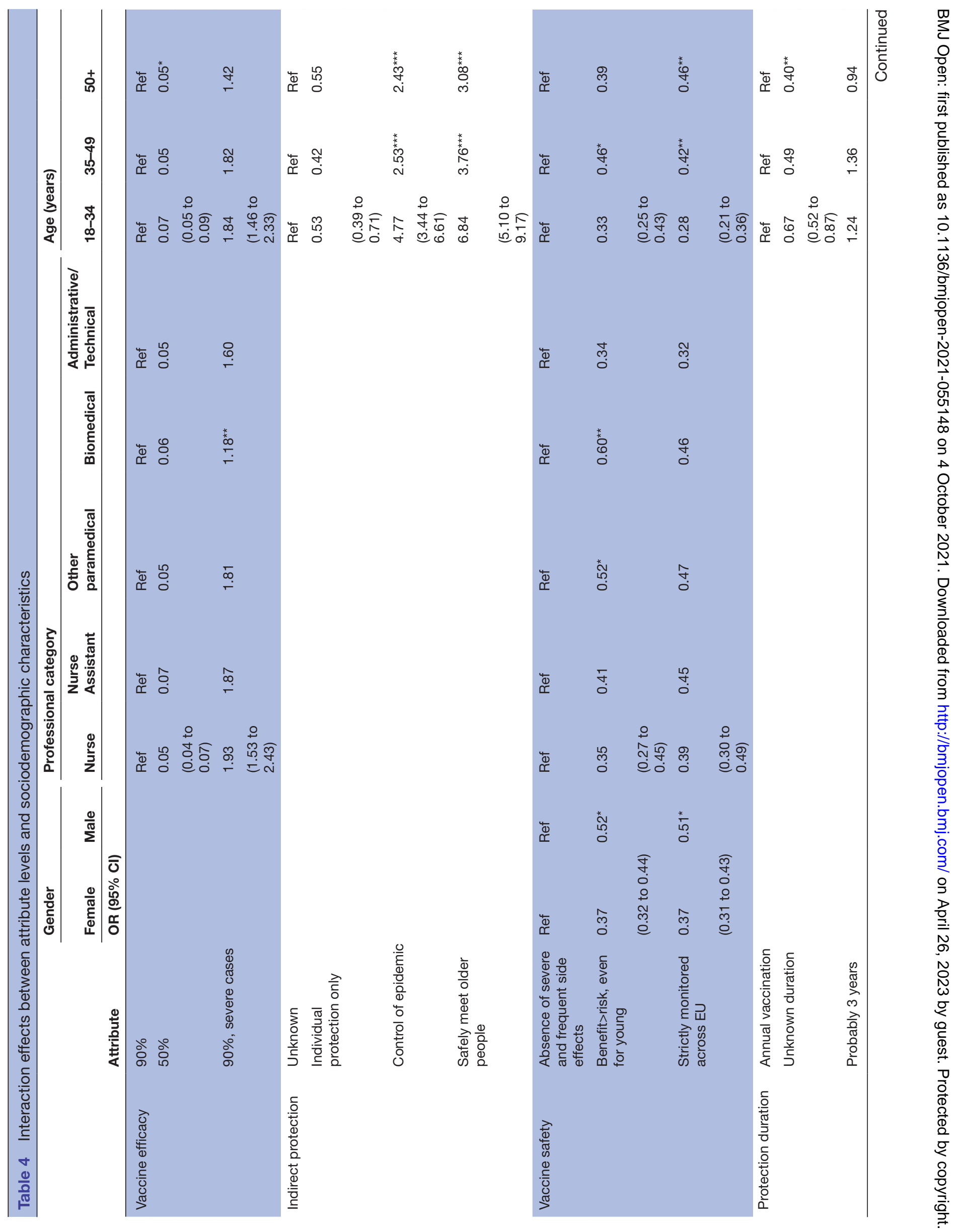



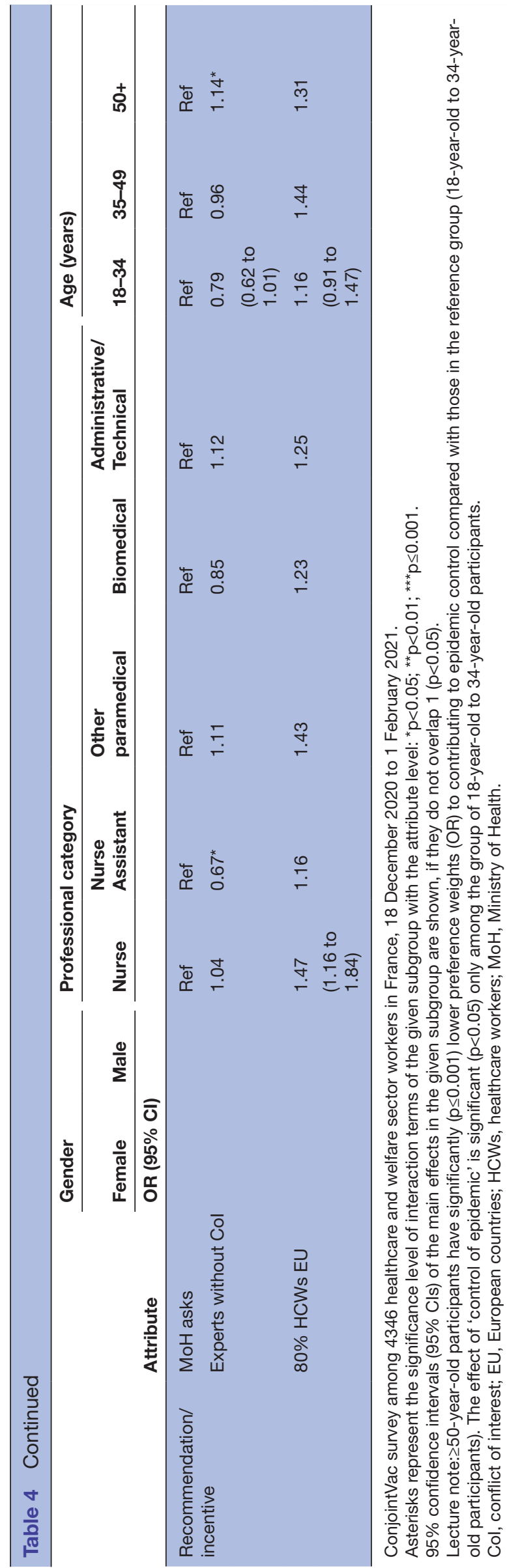

the healthcare and welfare system, play a role as trusted sources of health information, which emphasises the need to improve vaccine attitudes in these professional groups. The finding of a strong preference against COVID-19 vaccines with limited efficacy is consistent with previous findings from DCEs among the general adult population. ${ }^{10131422}$ This point is important in the current context of ongoing genetic diversification of SARS-CoV-2, which may lead to reduced vaccine effectiveness, ${ }^{23}$ and consequently lower acceptance of COVID-19 vaccination. This preference also may contribute to explaining slow uptake of Astra Zeneca vaccine when it was recommended to HCWs below 50 years of age at the start of the French vaccination campaign in early 2021. Similarly, limited and variable effectiveness due to annual strain variations is among known barriers to influenza vaccine acceptance, and the recommendation of the most effective vaccines is of highest importance.

At the start of our study, evidence on vaccine effectiveness against asymptomatic infection had not been available, while a substantial (although not perfect) protective effect against infection and transmission is now established for mRNA vaccines among HCW. ${ }^{24}{ }^{25} \mathrm{HCWs}$ are at risk to transmit SARS-CoV-2 to their patients and private environment. Our current results suggest that indirect protection is an important motivator for COVID-19 vaccination among HWSW. In a similar study on seasonal influenza vaccine among French $\mathrm{HCW},{ }^{18}$ we had found that contributing to disease control, protecting family members and protecting patients all substantially increased hypothetical willingness to accept influenza vaccination. Our results suggest that underpinning effectiveness against any infection and resulting protection of the social environment need to accompany reports on high clinical efficacy. Moreover, our results show that mentioning uncertainty about the vaccine effectiveness against infection and transmission was preferable to stating that there is none, which is of importance for upcoming COVID-19 vaccines. Using the approach of vaccine eagerness (derived from certainty in decision), we had the particular opportunity to observe that HWSWs who consistently refused hypothetical vaccination did have positive preferences towards indirect protection effects, in form of safe contacts with others and potential for epidemic control, similar to HWSWs with hesitating choices. Interestingly, these two attribute levels on collective benefits do create personal utility and represent altruism under its form of sympathy, rather than commitment. ${ }^{26}$ This interpretation may explain the seeming incoherence of our results with a randomised controlled trial on communication towards adults of the general population with strong hesitancy against COVID-19 vaccination. This trial found significant positive effects on a vaccine hesitancy score from mention of personal benefits, but not from collective benefits. ${ }^{27}$

We proposed three formulations of positive communication on vaccine safety, referring to the fact that the vaccines' safety profiles were under evaluation and only short-term observations were available ( 3 months at the 
time of the study). A clear risk negation was the preferable communication, even though the wording did not imply the absence of rare severe side effects. Similar to findings among French adolescents, ${ }^{21}$ the reference to a positive benefit-risk balance was demotivating, while this effect was weaker among biomedical professionals. The wording 'benefit-risk' itself implies the confirmation of a risk, and people may prefer negative consequences from omitting vaccination to negative consequences due to chosing vaccination. ${ }^{28}$ This is also known as prospect theory or loss aversion, which posits that losses (eg, health loss due to vaccine side effects) have more impact on individual decision-making than comparable gains. ${ }^{29} \mathrm{On}$ the other hand, information on the benefit-risk ratio is held imperative for the public to make an informed decision. $^{30}$ Similarly, the finding of the parallel knowledge and attitude evaluation in our survey, where a confident attitude towards the vaccine's benefit-risk ratio was the single strongest predictor of COVID-19 vaccine intention (personal communication coauthor JM). Further research is needed to explore how this benefit-risk notion can be translated into a more acceptable concept for communication, for example, utilising decision aids to reduce decisional conflict. ${ }^{31}$

Duration of vaccine protection was not a key attribute, possibly as HCWs are used to annual influenza vaccination. However, uncertainty around it was demotivating and long-term protection became important in scenarios with the potential of epidemic control through vaccination. This may reflect strategic collective thinking by participants. Sources of recommendation did not substantially affect vaccine acceptance either, except for a demotivating effect from experts even if they are without conflict of interest, specifically among nurse assistants and a motivating effect from the sample attribute level among serial non-demanders. Finally, the positive impact of mentioning a high coverage among HCWs in other EU countries illustrates the importance of social conformism, which has been described in other vaccine DCE for influenza vaccination among HCWs ${ }^{18}$ and COVID-19 among $\mathrm{HCWs}^{13}$ and in the general population ${ }^{10}$ and appeared strongly in the parallel knowledge and attitudes evaluation (personal communication coauthor JM).

We identified little heterogeneity of preferences across subgroups, which suggests that the recommendations emerging from our results can be applied to HWSWs in general, without risk of negative impact in a specific subgroup. Our study's main information carries on persons who may accept or refuse vaccination according to its characteristics. We could predict future uptake of COVID-19 vaccination in this group: almost complete with mRNA vaccines, low with vectorbased vaccines recommended to $\geq 55$-year-old persons and moderate for future vaccines with recent licensure and uncertainty about key characteristics. The great differences between professional categories underline the importance of targeted communication. Beyond this, recommending COVID-19 vaccines with limited effectiveness against severe disease and infectionalthough positive benefit-risk balance-will not meet HWSWs' preferences and yield low uptake.

\section{Study limitations}

Our study had several limitations. First, the snowball method of participant recruitment may encompass selection bias. The coverage with seasonal influenza vaccine in our sample $(51.9 \%)$ suggests that our participants were more favourable to vaccination compared with the HCW population in France (influenza coverage estimated at $35 \%) .{ }^{1}$ This is also illustrated by the high percentage of participants who uniformly chose to accept vaccination. We, therefore, cannot provide valid prevalence estimates for HWSW in France, only investigate attribute impacts on preferences and associations. However, the exploration of subgroups and results from vaccine eagerness among serial non-demanders suggest that the observed preference estimates may apply to the entire HWSW population. Furthermore, preference studies are known to overestimate willingness, ${ }^{32}$ and the stated preferences observed among participants in imaginary scenarios do not necessarily represent real-life choices. Finally, our DCE study was designed to evaluate the situation at the start of the COVID-19 vaccine campaign. Preferences may evolve over time as new data and vaccines emerge and other attributes may become more important to evaluate. However, preferences appeared relatively stable during the study period. Although our results are specific to French HWSWs and COVID-19 vaccination, they likely can optimise communication towards HCWs and the wider group of HWSWs in other countries, as the themes of vaccine hesitancy that are essential in optimised communication (in particular, complacency, lack of confidence, collective responsibility) have been described as factors of vaccine acceptance at international level and for other vaccinations. ${ }^{78}$

\section{CONCLUSION}

Despite these limitations, our study points towards several elements of improved COVID-19 vaccination promotion for HWSWs, in particular, those who are hesitating to get vaccinated: selecting vaccines with high efficacy and insisting on high effectiveness against severe disease; selecting vaccines for HWSWs that substantially reduce infection or transmission and insisting on consequences for social everyday life; avoiding the notion of 'risk-benefit balance' but provide information on both benefits and risks. HCW and more so HWSW in France are a heterogeneous group with different levels of higher education, vaccine hesitancy and risk exposure. Their preferences around vaccination may apply to some degree to the general population, in particular, items that reach beyond specific professional aspects and knowledge.

These results will be useful to inform vaccine promotion strategies and may help to develop adapted vaccine 
recommendations for HWSW as the vaccine response against the COVID-19 epidemic will most likely turn into a long-term vaccination strategy.

\section{Author affiliations}

${ }^{1}$ Department of Quantitative Methods in Public Health, EHESP French School of Public Health, Paris and Rennes, La Plaine St Denis, France

${ }^{2}$ LIRAES (EA4470), University of Paris, Paris, France

${ }^{3}$ Research Group for the Prevention of Occupational Infections in Healthcare Workers (GERES), Paris, France

${ }^{4}$ Emerging Infectious Disease Epidemiology Unit, Institut Pasteur, Paris, France ${ }^{5}$ Prevention and Health Promotion, Santé publique France, Saint-Maurice, France ${ }^{6}$ Department of Infectious Diseases, University Hopital of Saint-Etienne, SaintEtienne, France

${ }^{7}$ Chaire PreVacCl, Institut PRESAGE, University Jean Monnet University, Lyon University, Saint-Etienne, France

Acknowledgements We thank the GERES network members, U.R.P.S. Auvergne Rhône-Alpes and Union Française pour la santé bucco dentaire for distribution of the study invitation.

Contributors Coauthors have contributed as follows: Study design: JS, GP, SC, PA, CO, AG-B, EB-N, EB, JM. Funding acquisition: GP, PA, EB. Data collection: GP, PA, CO, AG-B, EB-N, EB. Data analysis: CDL, JM. Manuscript drafting: CDL, JS, JM. Manuscript review: all authors have reviewed the manuscript and validated the final version

Funding This work was supported by the French Public Health Agency (Santé Publique France).

Competing interests None declared.

Patient consent for publication Not applicable.

Ethics approval The study protocol was approved by the IRB of CHU St Etienne $\left(\mathrm{N}^{\circ}\right.$ IRBN1092021/CHUSTE) and the database was registered by EHESP French School of Public Health according to the GRDP regulation. Because the data collection was observational, collected no sensitive and only self-declared biomedical information, no informed consent was required. Participants visiting the study website saw the complete study information and agreed to study participation before starting the questionnaire. Study participation was anonymous without any risk of indirect identification. The funding source did not have any role in the conduct of the study or decision to submit this article.

Provenance and peer review Not commissioned; externally peer reviewed.

Data availability statement Data are available upon reasonable request.

Supplemental material This content has been supplied by the author(s). It has not been vetted by BMJ Publishing Group Limited (BMJ) and may not have been peer-reviewed. Any opinions or recommendations discussed are solely those of the author(s) and are not endorsed by BMJ. BMJ disclaims all liability and responsibility arising from any reliance placed on the content. Where the content includes any translated material, BMJ does not warrant the accuracy and reliability of the translations (including but not limited to local regulations, clinical guidelines, terminology, drug names and drug dosages), and is not responsible for any error and/or omissions arising from translation and adaptation or otherwise.

Open access This is an open access article distributed in accordance with the Creative Commons Attribution Non Commercial (CC BY-NC 4.0) license, which permits others to distribute, remix, adapt, build upon this work non-commercially, and license their derivative works on different terms, provided the original work is properly cited, appropriate credit is given, any changes made indicated, and the use is non-commercial. See: http://creativecommons.org/licenses/by-nc/4.0/.

ORCID iD

Judith Mueller http://orcid.org/0000-0003-0797-9971

\section{REFERENCES}

1 Santé Publique France, Bulletin de santé publique, Édition nationale. Couverture vaccinale antigrippale CheZ les professionnels de santé, 2019. Available: https://www.santepubliquefrance.fr/determinantsde-sante/vaccination/documents/bulletin-national/bulletin-de-santepublique-vaccination.-octobre-2019
2 Guthmann J, Abiteboul D. Vaccinations CheZ les soignants des établissements de soins de France, 2009. Couverture vaccinale, connaissances et perceptions vis- -vis des vaccinations. Rapport final. Santé Publique France; 76, 2011. Available: https://www. santepubliquefrance.fr/determinants-de-sante/vaccination/ documents/rapport-synthese/vaccinations-chez-les-soignants-desetablissements-de-soins-de-france-2009.-couverture-vaccinaleconnaissances-et-perceptions-vis-a-vis-des-vacci

3 Mueller JE, Olivier C, Diaz Luevano C. Cross-Sectional study on the seasonal flu and COVID-19 vaccination intentions of healthcare professionals: which levers for vaccine promotion? Bulletin Epidémiologique Hebdomadaire Covid-19 2021:2-9.

4 Santé Publique France. Point épidémiologique Du 8 juillet, 2021. Available: https://www.santepubliquefrance.fr/recherche/\# search=COVID\%2019\%20\%20\%20point\%20epidemiologique\& publications=donn\%C3\%A9es\&regions=National\&sort=date

5 Santé Publique France. Couverture vaccinale contre La COVID-19 CheZ les professionnels exerçant en établissements de santé, 2021. Available: https://www.santepubliquefrance.fr/content/download/ 366946/3126335

6 Gagneux-Brunon A, Detoc M, Bruel S, et al. Intention to get vaccinations against COVID-19 in French healthcare workers during the first pandemic wave: a cross-sectional survey. Journal of Hospital Infection 2021;108:168-73.

7 Crawshaw J, Konnyu K, Castillo G. Factors affecting healthcare worker COVID-19 vaccination acceptance and uptake: a living behavioural science evidence synthesis (V2, may 18th, 2021), 2021. Available: https://www.mcmasterforum.org/docs/default-source/ product-documents/living-evidence-syntheses/hcw-vaccinationliving-behavioural-science-evidence-synthesis-v2-(may-18).pdf? sfvrsn=8668fbc9 8

8 MacDonald NE, SAGE Working Group on Vaccine Hesitancy. Vaccine hesitancy: definition, scope and determinants. Vaccine 2015;33:4161-4.

9 Schwarzinger M, Watson V, Arwidson P, et al. COVID-19 vaccine hesitancy in a representative working-age population in France: a survey experiment based on vaccine characteristics. The Lancet Public Health 2021;6:e210-21.

10 Leng A, Maitland E, Wang S, et al. Individual preferences for COVID-19 vaccination in China. Vaccine 2021;39:247-54

11 Dong D, Xu RH, Wong EL-Y, et al. Public preference for COVID-19 vaccines in China: a discrete choice experiment. Health Expect 2020;23:1543-78.

12 Craig BM. United States COVID-19 vaccination preferences (CVP): 2020 hindsight. Patient 2021;14:309-18.

13 Fu C, Wei Z, Pei S. Acceptance and preference for COVID-19 vaccination in health-care workers (HCWs). medRxiv 2020.

14 Motta M. Can a COVID-19 vaccine live up to Americans expectations? A conjoint analysis of how vaccine characteristics influence vaccination intentions. Soc Sci Med 2021;272:113642.

15 Borriello A, Master D, Pellegrini A, et al. Preferences for a COVID-19 vaccine in Australia. Vaccine 2021;39:473-9.

16 McPhedran R, Toombs B. Efficacy or delivery? an online discrete choice experiment to explore preferences for COVID-19 vaccines in the UK. Econ Lett 2021;200:109747.

17 Bridges JFP, Hauber AB, Marshall D, et al. Conjoint analysis applications in Health-a checklist: a report of the ISPOR good research practices for conjoint analysis Task force. Value in Health 2011;14:403-13.

18 Godinot LD, Sicsic J, Lachatre M, et al. Quantifying preferences around vaccination against frequent, mild disease with risk for vulnerable persons: a discrete choice experiment among French Hospital health care workers. Vaccine 2021;39:805-14.

19 Peretti-Watel P, Seror V, Cortaredona S, et al. A future vaccination campaign against COVID-19 at risk of vaccine hesitancy and politicisation. Lancet Infect Dis 2020;20:769-70.

20 Lancsar E, Louviere J. Conducting discrete choice experiments to inform healthcare decision making. Pharmacoeconomics 2008;26:661-77.

21 Chyderiotis S, Sicsic J, Raude J, et al. Optimising HPV vaccination communication to adolescents: a discrete choice experiment. Vaccine 2021;39:3916-25

22 Kreps SE, Kriner DL. Factors influencing Covid-19 vaccine acceptance across subgroups in the United States: evidence from a conjoint experiment. Vaccine 2021;39:3250-8.

23 Madhi SA, Baillie V, Cutland CL, et al. Efficacy of the ChAdOx1 nCoV-19 Covid-19 vaccine against the B.1.351 variant. N Engl J Med 2021;384:1885-98.

24 Angel Y, Spitzer A, Henig O, et al. Association between vaccination with BNT162b2 and incidence of symptomatic and asymptomatic SARSCoV-2 infections among health care workers. JAMA 2021;325:2457. 
25 Shah AS, Gribben C, Bishop J. Effect of vaccination on transmission of COVID-19: an observational study in healthcare workers and their households. medRxiv 2021.

26 Shim E, Chapman GB, Townsend JP, et al. The influence of altruism on influenza vaccination decisions. J $R$ Soc Interface 2012;9:2234-43.

27 Freeman D, Loe BS, Yu L-M, et al. Effects of different types of written vaccination information on COVID-19 vaccine hesitancy in the UK (OCEANS-III): a single-blind, parallel-group, randomised controlled trial. Lancet Public Health 2021;6:e416-27.

28 Asch DA, Baron J, Hershey JC, et al. Omission bias and pertussis vaccination. Med Decis Making 1994;14:118-23.
29 Kahneman D, Tversky A. Prospect theory: an analysis of decision under risk. Econometrica 1979;47:263.

30 Bégué P. [Vaccine refusal and implications for public health in 2012]. Bull Acad Natl Med 2012;196:603-17.

31 Saunier F, Berthelot P, Mottet-Auselo B, et al. Impact of a decision-aid tool on influenza vaccine coverage among HCW in two French hospitals: a cluster-randomized trial. Vaccine 2020;38:5759-63.

32 Lehmann BA, Ruiter RAC, Chapman G, et al. The intention to get vaccinated against influenza and actual vaccination uptake of Dutch healthcare personnel. Vaccine 2014;32:6986-91. 